\title{
Implicaciones de las TIC en los derechos de autor
}

\author{
Raquel Cruz Coronel, Carlos David Cuesta Mundo, Jesús \\ Francisco García Pérez, Javier Guadalupe Martínez, Luis Enrique \\ Luna Martínez, Marinelsy Rosario Sierra, Scarlet Tejeda Pérez y \\ Martha Urrutia Ramírez
}

\section{Resumen}

Las Tecnologías de Información y Comunicación (TIC) son un conjunto de herramientas tecnológicas, electrónicas y digitales que, desde mediados del siglo xx, han mejorado la comunicación y la gestión de la información. Un conjunto de productos de almacenamiento, procesamiento y transmisión rápida y sencilla de información o datos que posibilitan la comunicación a largas distancias de manera multidireccional y muchas veces impersonal.

Por otro lado, los "derechos de autor" son prerrogativas legales que determinan privilegios y obligaciones de autores y derechohabientes sobre las creaciones literarias, arquitectónicas, artísticas, musicales, escultóricas, fotográficas, etcétera. Estas se dividen en "derechos morales" (derecho de los autores a decidir sobre la modificación, publicación, reproducción e integridad de sus obras) y los "derechos patrimoniales" (goce de las regalías que surjan del uso comercial, publicación, reproducción, traducción, adaptación, ejecución o trasmisión de las obras).

LaS Tıc han modificado la generación, representación, distribución y recuperación de la información. Los derechos de autor se han enfrentado a problemas respecto a la regulación de los procesos de reproducción, distribución y comunicación pública. El derecho de modificación, reproducción y distribución se ven vulnerados dadas las características de reproducción, gestión y transmisión de la información digital en nuestros días.

Palabras clave: derecho de autor; propiedad intelectual; tecnologías de la Información y comunicación; TIC; información. 


\title{
IMPLICATIONS OF ICT IN COPYRIGHT
}

\begin{abstract}
Information and Communication Technologies (ICT) are a set of technological, electronic and digital tools that, since the mid-20th century, have improved communication and information management. A set of products for storage, processing and fast and simple transmission of information or data that enable communication over long distances in a multidirectional and often impersonal manner.

On the other hand, "copyright" are legal prerogatives that determine the privileges and obligations of authors and rights holders over literary, architectural, artistic, musical, sculptural, photographic creations, et cetera. These are divided into "moral rights" (right of the authors to decide on the modification, publication, reproduction and integrity of their works) and the "patrimonial rights" (payment of the royalties arising from the commercial use, publication, reproduction, translation, adaptation, execution or transmission of works).

ICT have modified the generation, representation, distribution and retrieval of information. Copyright has faced problems regarding the regulation of reproduction, distribution and public communication processes. The right of modification, reproduction and distribution are violated given the characteristics of reproduction, management and transmission of digital information in our days.
\end{abstract}

Keywords: copyright; intellectual property; information and communication technologies; ICT; information.

\section{Raquel Cruz Coronel}

raquelhuapango@hotmail.com

Licenciada en Bibliotecología por la Facultad de Filosofía y Letras de la UNAM. Estudiante del 40 semestre de Maestría en Bibliotecología y Estudios de la Información. UNAM. Su tema de investigación es el Control de autoridad para autores personales con RDA. Actualmente labora como Bibliotecario en la sección de Análisis bibliográfico de la Universidad Autónoma Metropolitana, Unidad Iztapalapa, realizando actividades de catalogación de materiales bibliográficos, Discos Compactos y materiales audiovisuales, mediante las Reglas de Catalogación Angloamericanas (RCAA2), el sistema automatizado para Bibliotecas ALEPH en su versión 22 y el formato MARC21, así como actividades de clasificación, a través del Sistema de Clasificación de la Biblioteca del Congreso de los Estados Unidos (LC).

\section{Carlos David Cuesta Mundo}

davidcuesta@live.com.mx

Licenciado en Bibliotecología y Estudios de la Información y pasante de la licenciatura en Historia y de la Maestría en Bibliotecología y Estudios de la Información por la Universidad Nacional Autónoma de México. Sus líneas de investigación son: el acceso a la información documental, Bibliografía mexicana y la Historia de los movimientos sociales en México y Latinoamérica en el siglo XX. Actualmente apoya en las actividades del Área de Conservación y Documentación Sonora de la Fonoteca Nacional de México. 
Doctor en Bibliotecología y Estudios de la Información por la Universidad Nacional Autónoma de México y adscripto al Instituto de Investigaciones Sociales de la UNAM. Profesor en la Maestría en Bibliotecología y Estudios de la Información. Sus líneas de investigación son Propiedad intelectual, Derechos de autor, desarrollo de colecciones, industria editorial y el impacto del meme en las bibliotecas.

Javier Guadalupe Martínez

njosnavelin@comunidad.unam.mx

Licenciado en Bibliotecología y Estudios de la información y actual estudiante de la Maestría en misma disciplina en la Universidad Nacional Autónoma de México. Su línea de investigación es la Historia del libro y las bibliotecas. Ha sido invitado a exponer su tema de tesis de licenciatura en la clase se Historia del libro y las bibliotecas que se imparte en el Colegio de Bibliotecología en la Facultad de Filosofía y Letras de la UNAM, dada su rama especializada en las bibliotecas barrocas. Recientemente realizó una estancia de investigación en la Universidad de Salamanca en España, para obtener aportes al actual trabajo que desarrolla para su tesis de Maestría.

\section{Luis Enrique Luna Martínez}

Iwyzlunamar@gmail.com

Licenciado en Bibliotecología y Estudios de la información y actual estudiante de la Maestría en misma disciplina en la Universidad Nacional Autónoma de México.

\section{Marinelsy Rosario Sierra}

mary@habilisoluciones.com

Licenciada en Ciencias de la Información por la Universidad de La Habana y Maestra en Bibliotecología y Estudios de la Información por la Universidad Nacional Autónoma de México. Actualmente colabora con la empresa de ciencia, tecnología e información Habilis I \& CC. Sus líneas de investigación son la bibliometría, altmetrics, evaluación de la actividad científica.

\section{Scarlet Tejeda Pérez}

scarlet.tejeda22@gmail.com

Licenciada en Ciencias de la Información por la Universidad de La Habana y Maestra en Bibliotecología y Estudios de la Información por la Universidad Nacional Autónoma de México. Sus líneas de investigación son: la evaluación web, estudios métricos de la Información, edición de revistas científicas y gestión de la Información.

\section{Martha Urrutia Ramírez}

martha.urrutia.mu@gmail.com

Licenciada y Maestra en Bibliotecología y Estudios de la Información. Sus temas de investigación son: la música como fuente de información, preservación de archivos sonoros, la lectura y la formación lectora. 
Video. Clic para ver el video.

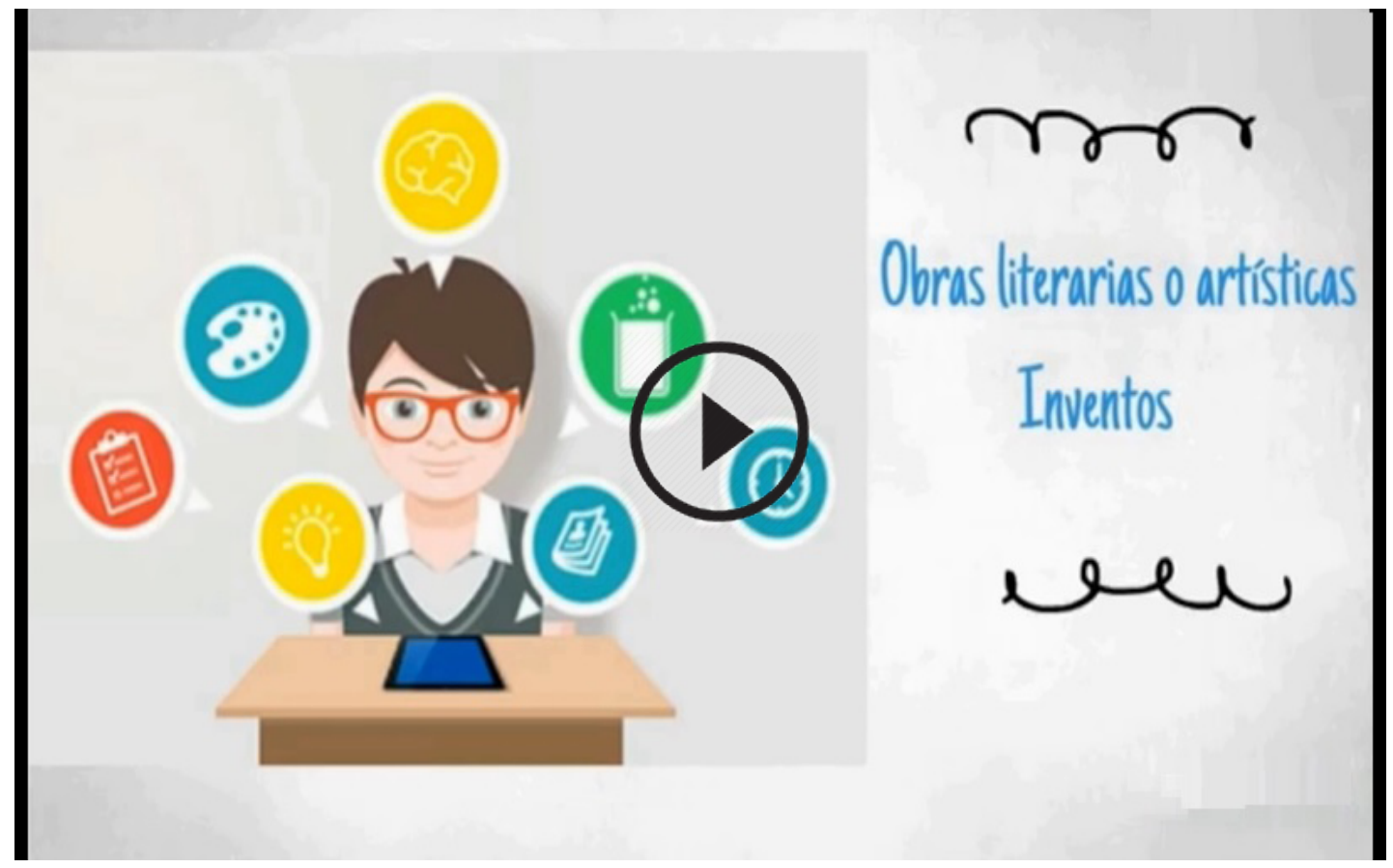

\section{Cómo citar este artículo}

* Cruz Coronel, R., Cuesta Mundo, C.D., García Pérez, J.F., Martínez, J.G., Luna Martínez, L.E., Rosario Sierra, M., Tejeda Pérez, S. y Urrutia Ramírez, M. (2019). Implicaciones de las TIC en los derechos de autor. Revista Digital Universitaria (RDU). Vol. 20, núm. 2 marzo-abril. Dol: http://doi.org/10.22201/codeic.16076079e.2019. v20n2.a9 